\title{
Surveillance des accidents vasculaires cérébraux au Manitoba, Canada : Estimations fondées sur des bases de données administratives
}

\author{
DF Moore, M.D., Ph.D. (1); LM Lix, Ph.D. (2); MS Yogendran, M.Sc. (3); P Martens, Ph.D. (3); A Tamayo, M.D. (4)
}

\section{Résumé}

Dans la présente étude, nous nous sommes penchés sur l'utilisation des bases de données administratives en matière de population dans la surveillance des accidents vasculaires cérébraux. Nous avons tout d'abord effectué une méta-analyse de quatre études, relevées au moyen d'une recherche dans PubMed, qui visaient à évaluer la sensibilité et la spécificité des données des hôpitaux dans la détermination des cas d'accident vasculaire cérébral lorsque les registres cliniques ou les dossiers médicaux étaient la méthode de référence. Nous avons ensuite mis au point des algorithmes de détermination des cas fondés sur les données des hôpitaux ainsi que les données relatives aux médecins et aux médicaments de prescription. Enfin, nous avons appliqué ces algorithmes aux données administratives du Manitoba, ce qui a permis d'obtenir des estimations de la prévalence pour les années financières 1995-1996 à 2003-2004, par groupe d'âge, sexe, région de résidence et quintile de revenu. Les résultats de la méta-analyse ont fait ressortir une certaine surdétermination des cas d'accident vasculaire cérébral à partir des données des hôpitaux, lorsque l'algorithme était fondé sur les codes de diagnostic applicables à n'importe quel type de maladie vasculaire cérébrale (rapport de cotes de Mantel-Haenszel [RC] $=1,70$ [intervalle de confiance (IC) à $95 \%: 1,53-1,88]$ ). Les analyses des données administratives du Manitoba ont révélé que même si le nombre total de cas d'accident vasculaire cérébral variait considérablement selon l'algorithme, la tendance de la prévalence était stable indépendamment de l'algorithme utilisé.

\section{Mots clés : données administratives, surveillance, santé de la population, accident vasculaire cérébral, longitudinal, diagnostics}

\section{Introduction}

L'accident vasculaire cérébral (AVC) se classe au troisième rang, après les cardiopathies et le cancer, parmi les causes de mortalité au Canada et dans les autres pays industrialisés; on estime que le fardeau et le taux de létalité liés à l'accident vasculaire cérébral surpassent ceux des autres maladies chroniques ${ }^{1-3}$. Malgré les progrès réalisés dans le domaine du traitement de l'accident vasculaire cérébral (AVC) aigu, la prévention des facteurs de risque liés à l'AVC demeurera sans doute le moyen le plus efficace de réduire le fardeau de la maladie ${ }^{4}$. La surveillance en matière de population permet aux chercheurs et aux analystes des politiques de décrire le fardeau de la maladie dans des groupes de population définis, en fonction de caractéristiques comme l'âge, le sexe, la région de résidence et le niveau de revenu. La surveillance peut aussi faciliter les évaluations de l'efficacité des stratégies de prévention du risque au fil du temps. Les vastes bases de données administratives ont été utilisées à des fins de surveillance en matière de population de plusieurs maladies chroniques, dont l'accident vasculaire cérébral ${ }^{5,6}$. Les données administratives présentent notamment les avantages suivants : a) capacité d'appliquer les estimations de la prévalence à l'ensemble de la population plutôt qu'à des sous-populations précises seulement, b) faibles coûts associés à l'établissement et au maintien d'un système de surveillance ${ }^{7}$, c) capacité de suivre les tendances de la prévalence et d) possibilité d'examiner des états comorbides connexes ${ }^{7}$.

Les données administratives des hôpitaux ont souvent servi à identifier les cas d'AVC dans la population, mais rares sont les études qui se sont intéressées à l'utilisation, à cette fin, des données relatives aux demandes de paiement des médecins ${ }^{8-12}$. Lors de la mise au point des algorithmes de détermination des cas au moyen de données administratives, le choix des codes de diagnostic à utiliser dans l'identification des cas d'AVC constituait une étape cruciale. Un algorithme fondé sur les codes diagnostiques visant toutes les formes de maladie vasculaire cérébrale aurait eu pour effet d'accroître la sensibilité, mais il aurait risqué d'entraîner une surdétermination des cas d'AVC, comparativement à une source de données cliniques. Un algorithme fondé sur un ensemble plus restreint de codes diagnostiques aurait permis d'accroître la spécificité, mais aurait pu donner lieu à des estimations prudentes du nombre de cas d'AVC ${ }^{13,14}$. Dans les publications médicales, l'AVC ischémique est souvent classé selon les critères du système TOAST (Trial of ORG 10172 in Acute Stroke Treatment) ${ }^{15-17}$. Ce système de classification permet de mieux définir l'étiologie de l'AVC

Coordonnées des auteurs

1 Walter Reed Army Medical Center, Washington, DC

2 School of Public Health, University of Saskatchewan, Saskatoon (Saskatchewan)

3 Centre manitobain des politiques en matière de santé, Département des sciences de la santé communautaire, Université du Manitoba (Manitoba)

4 Office régional de la santé de Brandon, Brandon (Manitoba)

Correspondance : Lisa M. Lix, University of Saskatchewan, Health Sciences Building, 107 Wiggins Road, Saskatoon (Saskatchewan) S7N 5E5, Tél. : 306-966-1617, Télec. : 306-966-7920,

Courriel : lisa.lix@usask.ca 
ischémique, en mettant l'accent sur les stratégies de traitement clinique, telles que l'administration de warfarine, dans les cas d'accident cardio-embolique, ou d'antiagrégants plaquettaires dans les cas d'affection des gros et des petits vaisseaux.

Pour l'instant, aucune étude n'a tenté d'élargir la méthode de détermination des cas d'AVC en incluant les données tirées des registres de délivrance de médicaments prescrits à la population ${ }^{7}$, qui sont maintenant systématiquement conservées dans un certain nombre de juridictions. Il se peut que la combinaison de ces trois sources de données - départs des hôpitaux, demandes de paiement des médecins et registres des médicaments de prescription - permette d'améliorer la détermination des cas d'AVC ${ }^{18,19}$. En fait, dans une étude portant sur six maladies chroniques (hypertension, insuffisance cardiaque, maladie pulmonaire chronique, arthrite, glaucome et diabète), le recours à plusieurs bases de données administratives dans la détermination des cas a permis d'atteindre une spécificité supérieure à 0,95 et une sensibilité dépassant 0,90 , comparativement à une source de données de validation indépendante ${ }^{20}$.

Dans la présente étude, nous avons tout d'abord eu recours à des techniques de méta-analyse pour évaluer la validité des diagnostics figurant dans les données administratives à des fins de détermination des cas d'AVC. Nous avons ensuite appliqué les résultats de la méta-analyse pour mettre au point des algorithmes de détermination des cas fondés sur les diagnostics consignés dans les données des hôpitaux et des médecins ainsi que dans les dossiers de délivrance des médicaments de prescription utilisés dans le traitement de l'AVC. Nous avons enfin appliqué les algorithmes aux données administratives du Manitoba (Canada), pour estimer la prévalence de l'AVC selon l'âge, le sexe, la région de résidence et le groupe de revenu au fil du temps.

\section{Méthodologie}

Méta-analyse de la détermination des cas d'accident vasculaire cérébral

Une recherche exhaustive dans PubMed à l'aide des termes administrative database (base de données administratives) ET stroke (accident vasculaire cérébral) OU cerebrovascular disease (maladie vasculaire cérébrale) a permis de relever en tout vingthuit articles pour la période 1965-2005. Après un examen approfondi de ces articles, effectué par l'auteur principal, quatre ont été retenus en vue d'un examen au moyen des techniques de méta-analyse. Toutes les études retenues avaient utilisé une " méthode de référence ", c'est-à-dire un registre des AVC tenu de manière indépendante ou un examen prospectif ou rétrospectif des dossiers, pour valider les données administratives à des fins de détermination des cas d'AVC. Les études retenues pour la méta-analyse reposaient sur des données d'hôpitaux dans lesquelles les diagnostics avaient été codés au moyen de la Classification internationale des maladies, 9e révision (CIM-9). Les études exclues de la méta-analyse n'avaient pas validé les données administratives, n'avaient pas eu recours aux codes de la CIM-9 pour déterminer les cas de maladie et/ou ne fournissaient pas les renseignements nécessaires à la création d'un tableau de classification 2 × 2 composé du nombre de cas et de non-cas d'AVC dans les ensembles de données administratives et de données de validation.

Dans les études retenues pour la métaanalyse, les cas d'AVC étaient identifiés au moyen d'un algorithme "sensible » fondé sur tous les codes diagnostiques de la maladie vasculaire cérébrale (c.-à-d. codes 430 à 438 de la CIM-9-MC) et/ou d'un algorithme "spécifique ", fondé sur un sous-ensemble de codes de la CIM-9-MC, lesquels sont les plus susceptibles d'identifier uniquement les cas d'AVC aigus à partir des données administratives.

Nous avons calculé les rapports de cotes (RC) pour chacune des études incluses dans la méta-analyse pour les algorithmes sensible et spécifique. Nous avons calculé les rapports de cotes groupés pour les algorithmes sensible et spécifique au moyen de la méthode de Mantel-Haenszel ${ }^{21,22}$. Les RC groupés ont été basés sur trois ensembles de données dans le cas de l'algorithme sensible, et sur quatre ensembles de données dans le cas de l'algorithme spécifique. Un $\mathrm{RC}=1,0$ indique que la probabilité d'un événement (c.-à-d. la détermination d'un cas d'AVC) est la même que l'on utilise les données administratives ou la source de données de validation. Un RC > 1,0 indique que les données administratives ont conduit à une surestimation du nombre de cas d'AVC comparativement à la source de données de validation; en revanche, un $\mathrm{RC}<1,0$ indique que le nombre de cas d'AVC est plus faible lorsqu'on utilise les données administratives plutôt que la source de données de validation. Pour effectuer la méta analyse, nous avons utilisé le logiciel SAS ${ }^{23}$.

\section{Détermination des cas d'AVC à partir des données administratives du Manitoba}

Pour estimer la prévalence de l'AVC avec les algorithmes de détermination des cas sensible et spécifique, nous avons eu recours au Dépôt de recherche, logé au Centre manitobain des politiques en matière de santé (CMPS). Le Dépôt de recherche a été utilisé dans de nombreuses études sur la santé de la population et l'utilisation des services de santé24,25.

Le CMPS conserve, relativement à toutes les personnes inscrites au registre de l'assurance-maladie du Manitoba, des données administratives des populations exhaustives, portant sur les départs des hôpitaux, les demandes de paiement des médecins et la délivrance des médicaments de prescription pour les patients non hospitalisés ${ }^{5}$. Selon les données de recensement de Statistique Canada, le Manitoba compte environ 1,2 million d'habitants ${ }^{26}$. Comme il n'y a pas de cotisation à payer, rares sont les personnes qui n'adhèrent pas au régime d'assurancemaladie $^{5}$. Il est possible de coupler, dans le temps, les fichiers de données administratives du Dépôt à l'aide d'un numéro d'identification personnelle de santé (NIPS) unique anonymisé. Les données démographiques relatives aux personnes inscrites à l'assurance-maladie, notamment l'âge, le sexe et le lieu géographique de résidence, peuvent être obtenues à partir du Dépôt, 
par couplage avec le registre de la population. Nous avons également pu déterminer les groupes de revenu en couplant les données du Dépôt avec celles relatives aux aires de diffusion. Ces dernières sont les plus petites régions géographiques pour lesquelles les données du recensement de Santé Canada sont diffusées.

Une feuille sommaire est remplie sur place au moment du départ d'un patient d'un établissement de soins actifs; chaque feuille sommaire contient jusqu'à 16 codes diagnostiques. Les demandes de paiement des médecins contiennent un seul code diagnostique. Au Manitoba, un petit nombre de médecins sont salariés; toutefois, la majorité d'entre eux soumettent une " demande de paiement pro forma » à des fins de facturation. On a estimé que les demandes de paiement pro forma rendent compte d'au moins $80 \%$ de ces services (Katz A., communication personnelle, février 2007). Dans les données des hôpitaux et des médecins, les diagnostics ont été consignés au moyen de la CIM-9 -MC jusqu'à l'année financière 2003-2004, mais à compter de l'année financière 2004-2005, on a introduit les codes de la CIM-10-CA dans les données de départ des hôpitaux.

Le Réseau pharmaceutique informatisé (RPI) est une base de données en ligne, au point de vente, sur les médicaments de prescription, qui relie toutes les pharmacies de détail au Manitoba. Le RPI enregistre la délivrance des médicaments de prescription pour tous les résidants du Manitoba, indépendamment du régime d'assurance dont ils bénéficient. Les médicaments de prescription sont identifiés au moyen des DIN (numéro d'identification du médicament), lesquels sont couplés avec la Base de données sur les produits pharmaceutiques (BDPP) de Santé Canada. Les codes du Système de classification anatomique, thérapeutique, chimique (ATC) ${ }^{27}$ sont ajoutés de façon à permettre le classement des médicaments dans les sous groupes thérapeutiques et pharmacologiques appropriés.

Nous avons effectué des recherches parmi les 16 champs diagnostiques figurant dans les feuilles sommaires de départ des hôpitaux pour relever les cas d'AVC. On trouve au tableau 1 les codes de la CIM-9MC correspondant à la maladie vasculaire cérébrale, le type d'AVC auquel chaque code correspond et sa relation avec les critères du système TOAST. Selon ce système, l'AVC est considéré comme un AVC lié à : a) l'athérosclérose d'une grosse artère (y compris la thrombose d'une grosse artère et l'embolie d'artère à artère), b) l'embolie cardiaque, c) l'occlusion d'une petite artère, d) un accident vasculaire cérébral attribuable à une autre cause définie et/ou e) un accident vasculaire cérébral de cause indéterminée. La catégorie accident cardioembolique du système TOAST ne peut être indiquée au moyen des codes de la CIM-9-MC.

Après avoir passé en revue la littérature et consulté des spécialistes cliniques, les auteurs ont retenu les catégories suivantes de médicaments dans l'identification des cas d'AVC à partir des données du RPI : a) les antiagrégants plaquettaires, tels que l'aspirine (AAS) à la dose de 81 ou $325 \mathrm{mg}$ une fois par jour, le clopidogrel, la ticlopidine, le dipyridamole et des associations médicamenteuses telles que Aggrenox

TABLEAU 1

Codes de la CIM-9-MC pour la détermination des cas d'accident vasculaire cérébral à partir des données administratives, relation avec les critères TOAST et fréquence dans les départs des hôpitaux au Manitoba, 1995-1996 à 2003-2004

\begin{tabular}{|c|c|c|c|c|}
\hline $\begin{array}{l}\text { Code de la } \\
\text { CIM-9-MC }\end{array}$ & Type d'AVC & Critères TOAST & Fréqu. & $\%$ \\
\hline 430 & Hémorragie sous arachnoïdienne & S.o. ${ }^{\mathrm{a}}$ & 901 & 1,7 \\
\hline 431 & Hémorragie cérébrale & s.o. & 2038 & 4,0 \\
\hline 432 & $\begin{array}{l}\text { Hémorragies intracrâniennes } \\
\text { autres et sans précision }\end{array}$ & s.o. & 951 & 1,8 \\
\hline 433 & $\begin{array}{l}\text { Occlusion et sténose } \\
\text { des artères précérébrales }\end{array}$ & Maladie des gros vaisseaux & 5957 & 11,9 \\
\hline 434 & Occlusion des artères cérébrales & $\begin{array}{l}\text { Maladie des gros et } \\
\text { des petits vaisseaux }\end{array}$ & 5968 & 11,9 \\
\hline 435 & Ischémie cérébrale transitoire & s.o. & 8189 & 16,3 \\
\hline 436 & $\begin{array}{l}\text { Maladies cérébro- vasculaires } \\
\text { aiguës mais mal définies }\end{array}$ & AVC - autre cause définie & 12061 & 24,0 \\
\hline 437 & $\begin{array}{l}\text { Maladies cérébro vasculaires, } \\
\text { autres et mal définies }\end{array}$ & AVC - cause indéterminée & 2667 & 6,5 \\
\hline 438 & $\begin{array}{l}\text { Séquelles des maladies } \\
\text { cérébro vasculaires }\end{array}$ & s.o. & 12266 & 22,4 \\
\hline Tous & & & 50098 & 100,0 \\
\hline
\end{tabular}

${ }^{\mathrm{a}}$ S.o. = sans objet.

b Les fréquences représentent le nombre de personnes (âgées de 19 ans et plus) ayant eu au moins un départ de l'hôpital qui a donné lieu au code indiqué de la CIM 9 MC.
(AAS 25 mg, dipyridamole $200 \mathrm{mg}$, à libération lente) b) les anticoagulants oraux, tels que la warfarine, la phénindione et la nicoumalone. Les codes ATC (cinquième niveau) étaient les suivants : B01AA02, B01AA03, B01AA07, B01AC07, B01AB01, B01AC30, B01AC05, B01AC06, B01AC04, B01AB09, B01AB04, B01AB10. Les agents thrombolytiques comme le rt-PA (activateur tissulaire du plasminogène recombinant) et les antiagrégants plaquettaires intraveineux (anti GP 2b/3a), comme l'abciximab, le tirofiban et l'eptifibatide, n'ont pu être identifiés parmi les données du RPI.

Nous avons utilisé les règles suivantes pour déterminer les cas d'AVC : au moins un départ de l'hôpital au cours d'une année financière (c.-à-d. H 1 +), ou au moins deux demandes de paiement d'un médecin pour le code de la CIM-9-MC (c.-à-d. M $2+$ ) au cours d'une année financière, ou au moins une demande de paiement d'un médecin au cours d'une année financière ainsi qu'au moins deux enregistrements de médicaments de prescription au cours d'une année financière (c.-à-d. M $1+$ et Rx $2+$ ). Nous avons calculé le nombre de cas pour chacune des années 
financières de 1995-1996 à 2003-2004. L'année financière correspond à la période allant du $1^{\text {er }}$ avril au 31 mars de l'année suivante. Nous avons choisi cette période parce que le système du RPI a été mis sur pied en 1994-1995, tandis que le codage au moyen de la CIM-10-CA a été introduit en 2004-2005. Pour garantir la cohérence, nous avons donc appliqué annuellement un algorithme de détermination des cas, fondé sur des codes de la CIM-9-MC.

La fréquence des cas d'AVC a été consignée, pour l'algorithme sensible et l'algorithme spécifique, par année d'étude, groupe d'âge (19 à 44, 45 à 54 ans, 55 à 64 ans, 65 à 74 ans, 75 à 84 ans, 85 ans et plus), sexe, région de résidence (offices régionaux de la santé du Nord du Manitoba, offices régionaux de la santé du Sud du Manitoba et office régional de la santé de Winnipeg l'office régional de la santé représentant l'unité sanitaire administrative de la province) et quintile de revenu (Q1 à Q5 , le Q1 représentant le groupe de revenu le plus faible). Les régions géographiques correspondent, de manière générale, à des collectivités rurales peu peuplées, à des collectivités rurales et à des collectivités urbaines. Pour déterminer le quintile de revenu des sujets, nous avons utilisé les données sur le revenu moyen des ménages pour les aires de diffusion, puis nous avons effectué un classement en fonction de ces aires. Les quintiles ont été définis de manière que chaque groupe soit constitué d'environ $20 \%$ de la population ${ }^{24}$ globale. Les quintiles de revenu sont définis séparément pour les régions urbaines et les régions rurales. Dans le calcul des estimations de la prévalence, nous avons utilisé les données du registre provincial pour établir le dénominateur de l'estimation.

Nous avons effectué des analyses de régression afin de relever les différences éventuelles dans le taux relatif (TR) d'AVC pour différents sous-groupes de population et dans le temps. Nous avons d'abord analysé les données relatives à chaque année d'étude au moyen de modèles linéaires généralisés, afin de relier le nombre d'AVC aux effets principaux des variables groupe d’âge, sexe, région de résidence, quintile de revenu et algorithme (c.-à-d. sensible et spécifique), ainsi qu’à certaines interactions bidirectionnelles parmi ces variables. Afin de garantir la parcimonie du modèle, nous avons retenu uniquement les interactions qui entraînaient une amélioration importante de l'ajustement du modèle, tel qu'évalué au moyen du test du rapport des vraisemblances maximales. Une valeur de correction, le logarithme de la population totale, a été intégrée dans tous les modèles. On a initialement utilisé des paramètres pour ces données à l'aide de la distribution de Poisson, de la distribution binomiale négative et de la distribution gamma. Nous avons comparé les statistiques de l'adéquation des différentes distributions et choisi celles permettant d'obtenir le modèle de meilleur ajustement. Les données longitudinales ont été analysées au moyen d'un modèle linéaire généralisé avec la méthode de l'équation d'estimation généralisée (EEG), afin de prendre en compte la corrélation parmi les nombres de cas d'AVC au fil du temps ${ }^{29}$. Les principaux effets du groupe d'âge, du sexe, de la région de résidence, du quintile de revenu, de l'algorithme et de l'année/ moment ont été inclus dans le modèle. Certaines interactions bidirectionnelles ont été incluses, mais ces effets du modèle n’ont été retenus que s’ils permettaient d'améliorer de façon importante l'ajustement du modèle. Encore une fois, on a initialement utilisé des paramètres pour ces données à l'aide de la distribution de Poisson, de la distribution binomiale

TABLEAU 2

\section{Rapports de cotes $(\mathrm{RC})$ de la méta-analyse portant sur la détermination des cas d'accident vasculaire cérébral à partir des données des hôpitaux, pour des ensembles sensible et spécifique de codes diagnostiques}

\begin{tabular}{lcc}
\hline Étude & $\begin{array}{c}\text { Algorithme sensible } \\
\text { RC (IC à 95 \%) }\end{array}$ & $\begin{array}{c}\text { Algorithme spécifique } \\
\text { RC (IC à 95 \%) }\end{array}$ \\
\hline Ellekjaer et coll. $^{32}$ & $1,76(1,51,2,05)$ & $1,17(1,00,1,38)$ \\
Leibson et coll. $^{18}$ & $1,47(1,17,1,86)$ & $1,20(0,95,1,53)$ \\
Reker et coll. $^{33}$ & $1,76(1,46,2,10)$ & $0,72(0,58,0,89)$ \\
Tirschwell et coll. $^{13}$ & - & $1,02(0,80,1,31)$ \\
\hline RC groupé & $1,70(1,53,1,88)$ & $1,02(0,93,1,13)$ \\
\hline
\end{tabular}

a L'algorithme sensible est fondé sur les codes 430 à 438 de la CIM-9-MC dans toutes les études, sauf celle de Reker et coll. ${ }^{33}$, qui a exclu les codes 437 et 438.

b L'algorithme spécifique est fondé sur les codes 430, 431, 434 et 436 de la CIM-9-MC dans toutes les études, sauf celle de Reker et coll. ${ }^{33}$ (code 436 exclu), de Leibson et coll. ${ }^{18}$ (code 437 inclus) et de Tirschwell et coll. ${ }^{13}$ (code 435 inclus). 
TABLEAU 3

Fréquence (\%) des cas d'accident vasculaire cérébral (AVC), selon la source de données et les codes de la CIM-9-MC et prévalence brute de l'AVC, 1995-1996 - 2003-2004

\begin{tabular}{|c|c|c|c|c|c|c|c|c|}
\hline \multirow{2}{*}{$\begin{array}{l}\text { Année } \\
\text { financière }\end{array}$} & \multicolumn{2}{|c|}{$\begin{array}{l}\text { Hôpitala }^{\mathbf{a}} \\
\text { (H } 1+\text { ) }\end{array}$} & \multicolumn{2}{|c|}{$\begin{array}{l}\text { Médecin } \\
\text { (M 2+) }\end{array}$} & \multicolumn{2}{|c|}{$\begin{array}{l}\text { Médecin + médicament } \\
\quad(\text { M } 1 \text { + et Rx } 2+)\end{array}$} & \multicolumn{2}{|c|}{ Total } \\
\hline & Fréqu. & $\%$ & Fréqu. & $\%$ & Fréqu. & $\%$ & Fréqu. & Prév. (\%) \\
\hline \multicolumn{9}{|c|}{ Algorithme sensible (codes 430 à 438 de la CIM-9-MC) } \\
\hline 1995-1996 & 4882 & 49,9 & 4349 & 44,5 & 551 & 5,6 & 9782 & 1,16 \\
\hline 1996-1997 & 5053 & 51,5 & 4203 & 42,9 & 547 & 5,6 & 9803 & 1,16 \\
\hline 1997-1998 & 4790 & 48,7 & 4339 & 44,1 & 701 & 7,1 & 9830 & 1,16 \\
\hline 1998-1999 & 4777 & 48,6 & 4357 & 44,3 & 702 & 7,1 & 9836 & 1,16 \\
\hline $1999-2000$ & 4488 & 45,8 & 4398 & 44,9 & 920 & 9,4 & 9806 & 1,16 \\
\hline $2000-2001$ & 4585 & 44,3 & 4587 & 44,3 & 1176 & 11,4 & 10348 & 1,21 \\
\hline 20012002 & 4276 & 41,5 & 4557 & 44,3 & 1462 & 14,2 & 10295 & 1,20 \\
\hline $2002-2003$ & 3948 & 39,9 & 4447 & 45,0 & 1488 & 15,1 & 9883 & 1,14 \\
\hline 20032004 & 3993 & 38,5 & 4746 & 45,7 & 1635 & 15,8 & 10374 & 1,19 \\
\hline \multicolumn{9}{|c|}{ Algorithme spécifique no 1 (codes 430, 431, 434, 435, 436 de la CIM-9-MC) } \\
\hline 1995-1996 & 3283 & 43,8 & 3704 & 49,4 & 517 & 6,9 & 7504 & 0,89 \\
\hline 1996-1997 & 3239 & 43,8 & 3573 & 48,3 & 584 & 7,9 & 7396 & 0,88 \\
\hline 1997-1998 & 3166 & 42,3 & 3656 & 48,9 & 662 & 8,8 & 7484 & 0,89 \\
\hline 1998-1999 & 3234 & 43,4 & 3554 & 47,7 & 656 & 8,8 & 7444 & 0,88 \\
\hline 1999-2000 & 2956 & 39,9 & 3603 & 48,6 & 855 & 11,5 & 7414 & 0,87 \\
\hline $2000-2001$ & 2955 & 37,5 & 3836 & 48,7 & 1084 & 13,8 & 7875 & 0,92 \\
\hline 2001-2002 & 2831 & 36,1 & 3726 & 47,5 & 1281 & 16,3 & 7838 & 0,91 \\
\hline $2002-2003$ & 2666 & 35,6 & 3546 & 47,4 & 1275 & 17,0 & 7487 & 0,87 \\
\hline 2003-2004 & 2705 & 33,8 & 3869 & 48,4 & 1422 & 17,8 & 7996 & 0,92 \\
\hline \multicolumn{9}{|c|}{ Algorithme spécifique $n^{\circ} 2$ (codes 430, 431, 434, 436 de la CIM-9-MC) } \\
\hline 1995-1996 & 2431 & 45,1 & 2518 & 46,7 & 439 & 8,1 & 5388 & 0,64 \\
\hline 1996-1997 & 2406 & 45,7 & 2433 & 46,2 & 426 & 8,1 & 5265 & 0,62 \\
\hline 1997-1998 & 2281 & 43,2 & 2525 & 47,9 & 468 & 8,9 & 5274 & 0,62 \\
\hline 1998-1999 & 2328 & 44,1 & 2478 & 46,9 & 475 & 9,0 & 5281 & 0,62 \\
\hline $1999-2000$ & 2091 & 40,1 & 2507 & 48,1 & 614 & 11,8 & 5212 & 0,61 \\
\hline $2000-2001$ & 2197 & 39,5 & 2599 & 46,8 & 761 & 13,7 & 5557 & 0,65 \\
\hline 2001-2002 & 2113 & 37,7 & 2601 & 46,3 & 898 & 16,0 & 5612 & 0,65 \\
\hline $2002-2003$ & 2035 & 37,8 & 2468 & 45,9 & 875 & 16,3 & 5378 & 0,62 \\
\hline 2003-2004 & 2023 & 35,3 & 2683 & 46,8 & 1024 & 17,9 & 5730 & 0,66 \\
\hline
\end{tabular}

a Dans la détermination des cas d'accident vasculaire cérébral, les départs des hôpitaux $(\mathrm{H} 1+$ ) priment sur les demandes de paiement des médecins (M $2+$ ), lesquelles priment sur les données combinées des médecins et des médicaments de prescription (M 1 + et Rx $2+$ ) dans l'identification des cas d'accident vasculaire cérébral.

Détermination des cas d'accident vasculaire cérébral à partir des données administratives du Manitoba

Le tableau 1 présente la fréquence des cas d'AVC relevés à partir des données administratives des hôpitaux pour chacun des codes 430 à 438 de la CIM-9-MC au cours de la période 1995-1996 à 2003-2004. Plus de la moitié des cas $(54,7 \%)$ étaient accompagnés des codes diagnostiques non spécifiques 432, 436, 437 et 438 .
Nous examinons ci-après la fréquence des cas d'AVC à partir des données administratives des hôpitaux, des médecins et des pharmacies. Nous avons d'abord relevé le nombre de cas satisfaisant au critère H 1 +, puis le nombre de cas satisfaisant au critère M 2 + et enfin le nombre de cas additionnels satisfaisant au critère M $1+$ et Rx $2+$. Les résultats sont déclarés séparément pour l'algorithme sensible basé sur tous les diagnostics de maladie vasculaire cérébrale figurant dans les données administratives des hôpitaux et des médecins, et pour l'algorithme spécifique basé sur un sous-ensemble de diagnostics les plus susceptibles de correspondre à des cas d'accident vasculaire cérébral aigu. Dans le dernier cas, nous avons présenté, initialement, deux sous-ensembles différents de codes de la CIM-9-MC : un ensemble comprenait les accidents ischémiques transitoires, l'autre non. Seuls les résultats du premier algorithme spécifique sont inclus dans les analyses de régression ultérieures. 
TABLEAU 4

Analyses de régression du taux relatif (TR) d’accident vasculaire cérébral au Manitoba, 1998-1999

\begin{tabular}{|c|c|c|}
\hline Effet du modèle & Estimation (ET) $)^{a, b}$ & TR (IC à $95 \%)^{c}$ \\
\hline Rég. rurale, Sud & $-0,27(0,05)$ & $0,77(0,70,0,84)$ \\
\hline Winnipeg & $-0,25(0,05)$ & $0,78(0,71,0.85)$ \\
\hline Rég. rurale, Nord & Réf & - \\
\hline 85 ans et plus & $4,54(0,05)$ & $93,41(84,92,102,76)$ \\
\hline $75-84$ ans & $4,24(0,05)$ & $69,61(63,67,76,10)$ \\
\hline $65-74$ ans & $3,55(0,05)$ & $34,97(31,95,38,28)$ \\
\hline $55-64$ ans & $2,72(0,05)$ & $15,20(13,83,16,72)$ \\
\hline $45-54$ ans & $1,70(0,05)$ & $4,47(4,93,6,06)$ \\
\hline $19-44$ ans & Réf & - \\
\hline Quintile 5 & $-0,30(0,03)$ & $0,74(0,69,0,79)$ \\
\hline Quintile 4 & $-0,20(0,03)$ & $0,82(0,49,0,62)$ \\
\hline Quintile 3 & $-0,16(0,03)$ & $0,86(0,81,0,91)$ \\
\hline Quintile 2 & $-0,13(0,03)$ & $0,88(0,83,0,93)$ \\
\hline Quintile 1 & Réf & - \\
\hline Hommes & $0,23(0,02)$ & $1,26(1,21,1,31)$ \\
\hline Femmes & Réf & - \\
\hline Algorithme spécifique ${ }^{d}$ & $-0,27(0,02)$ & $0,77(0,74,0,80)$ \\
\hline Algorithme sensible & Réf & - \\
\hline \multicolumn{3}{|l|}{ ET = erreur type. } \\
\hline \multicolumn{3}{|c|}{$\begin{array}{l}\text { ' Les estimations des paramètres ont été obtenues au moyen d'un modèle linéaire généralisé ayant une distribution } \\
\text { binomiale négative. }\end{array}$} \\
\hline \multicolumn{3}{|c|}{ ' IC = intervalle de confiance. } \\
\hline
\end{tabular}

Comme le montre le tableau 3 pour l'algorithme sensible, 49,9\% des cas d'AVC ont été relevés à partir des données des hôpitaux au début de la période d'étude (c.-à-d. 1995-1996); ce pourcentage a chuté de façon marquée à $38,5 \%$ à la fin de la période d'étude. Le pourcentage de cas relevés uniquement à partir des données des médecins est cependant demeuré relativement constant au fil du temps. Quant au pourcentage de cas relevés à la fois à partir des données des médecins et des données relatives aux médicaments de prescription, il a augmenté au fil du temps, passant de 5,6 \% en 1995-1996 à 15,8 \% en 2003-2004. L'utilisation de l'algorithme plus spécifique faisait ressortir la même tendance, c.-à-d. une diminution du nombre de cas d'AVC relevés à partir des données des hôpitaux et une augmentation du nombre de cas d'AVC relevés à partir des données des médecins et de celles relatives aux médicaments de prescription.

Les estimations brutes de la prévalence à l'échelle provinciale (tableau 3) sont demeurées relativement inchangées au fil du temps, indépendamment de l'algorithme utilisé. Toutefois, le taux fondé sur le plus petit ensemble de codes de la CIM-9-MC correspond à environ la moitié de la valeur du taux calculé en utilisant l'ensemble complet de codes diagnostiques relatifs à la maladie vasculaire cérébrale.

Nous avons ensuite analysé le nombre de cas d'AVC à chaque année, au moyen de modèles linéaires généralisés. La distribution binomiale négative permettait un meilleur ajustement des données que la distribution de Poisson ou la distribution gamma, comme en témoignait le rapport entre la déviance résiduelle et le degré de liberté des modèles. Le test du rapport de vraisemblance a révélé que les modèles contenant des interactions bidirectionnelles ne permettaient pas un ajustement des données nettement meilleur que celui obtenu avec un modèle plus simple, ne contenant que les effets principaux $(p>$ $0,05)$; c'est la raison pour laquelle le dernier modèle a été retenu. Les résultats du modèle pour l'année financière 1998-1999 sont présentés au tableau 4; nous avons observé des résultats semblables pour toutes les autres années, c'est pourquoi ces résultats ne sont pas présentés ici. Le taux relatif (TR) d'accident vasculaire cérébral était significativement plus bas dans les régions rurales et urbaines du Sud que dans le Nord du Manitoba et significativement plus élevé dans les groupes âgés. Nous avons observé un gradient de revenu, le TR de l'AVC étant plus faible dans les quintiles de revenu supérieurs. Le taux était plus élevé chez les hommes que chez les femmes, et il était significativement plus bas dans le cas de l'algorithme spécifique que dans celui de l'algorithme sensible.

Les données longitudinales de prévalence ont également été modélisées. Cette fois encore, nous avons choisi une distribution négative binomiale, parce qu'elle permettait un meilleur ajustement de ces données que la distribution gamma ou la distribution de Poisson. L'inclusion des termes d'interaction « année $\mathrm{x}$ région » et « année $\mathrm{x}$ groupe d'âge » a permis d'améliorer sensiblement l'ajustement du modèle; d'autres termes d'interaction bidirectionnelle n'ont pas été retenus pour le modèle parce qu'ils n'amélioraient pas de façon marquée l'ajustement. Pour l'interaction " année x région », les analyses ont révélé qu'après ajustement pour tenir compte des autres effets du modèle, le TR de changement dans la prévalence de l'AVC était supérieur dans les régions rurales (TR $=1,01$, IC à $95 \%=$ $1,00,1,02$ ) et les régions urbaines du Sud $(\mathrm{TR}=1,02$, IC à $95 \%=1,01,1,04)$ que dans les régions du Nord du Manitoba. Dans ce dernier cas, les analyses ont révélé que comparativement au groupe d'âge des 19 à 44 ans, le TR de changement dans la prévalence de l'AVC était plus faible dans le groupe des 55 à 64 ans (TR = 0,96; IC à $95 \%=0,95,0,99)$, des 65 à 74 ans (TR = 0,96; IC à $95 \%=0,94,0,97)$, des 75 à 84 ans (TR $=0,95$; IC à $95=0,94,0,97)$ et des 85 ans et plus (TR =0,96; IC à $95 \%=$ $0,94,0,98)$. Les figures 1 et 2 illustrent la nature des tendances dans les estimations de la prévalence pour les groupes d'âge et les régions. Les principaux effets des variables « sexe », " quintile de revenu » et " algorithme " étaient également significatifs $(p<0,05)$, et les estimations du TR étaient semblables à celles présentées au tableau 4. 


\section{FIGURE 1}

Tendances de la prévalence brute de l'accident vasculaire cérébral par groupe d'âge pour a) l'algorithme sensible et b) l'algorithme spécifiquea, 1995-1996 à 2003-2004
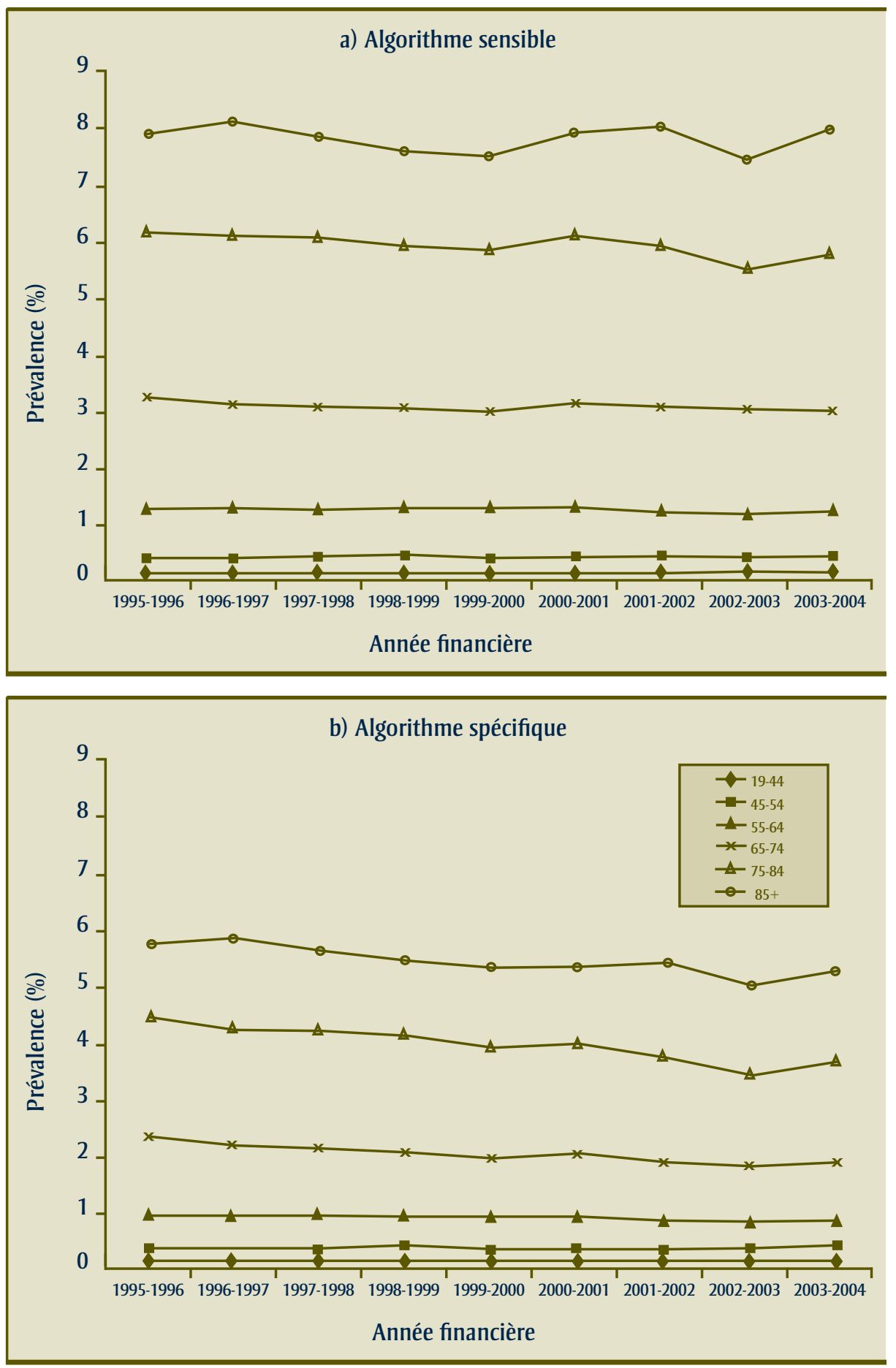

a L’algorithme spécifique est basé sur les codes suivants de la CIM-9-MC : 430, 431, 434, 435 et 436.

\section{Analyse}

Cette étude s'appuie sur des recherches antérieures qui se sont penchées sur le rôle potentiel des données administratives dans la surveillance des accidents vasculaires cérébraux. Un certain nombre d'algorithmes de détermination des cas ont été appliqués aux bases de données administratives existantes dans les provinces et territoires du Canada. Les estimations de la prévalence de l'AVC ont été indirectement validées au moyen d'une méta-analyse des études antérieures qui ont comparé les données des hôpitaux à celles des dossiers médicaux ou des registres. Les résultats de la métaanalyse ont révélé que la probabilité de concordance entre les données administratives et celles des dossiers ou des registres était meilleure lorsqu'on utilisait un ensemble spécifique de codes diagnostiques plutôt qu'un ensemble sensible de codes.

L'examen de la distribution des cas d'AVC parmi l'ensemble des codes diagnostiques peut aider à déterminer les besoins en matière de ressources liées aux soins de santé. Nous avons constaté qu'au Manitoba, parmi les données des hôpitaux, près de 1000 cas d'AVC par année (c.-à-d. environ $16 \%)$ sont identifiés comme étant des accidents ischémiques transitoires qui, par définition, ne sont pas associés à des déficits neurologiques fonctionnels étendus. Les patients qui en sont victimes se prêtent bien à la prévention secondaire, au moyen d'une évaluation rigoureuse des facteurs de risque vasculaire cérébral, suivie d'un traitement chirurgical ou médical. Nous avons observé que le nombre d'hémorragies sous-arachnoïdienne et cérébrale représente environ $10 \%$ de l'ensemble des cas d'AVC au Manitoba, par opposition au taux de $20 \%$ habituellement cité pour l'accident hémorragique cérébral dans la majorité des études de population. Il y a tout lieu de croire que le codage de l'AVC hémorragique est fiable, vu la nature de la consultation clinique à laquelle il donne lieu, toutefois, pour faire réellement la lumière sur ces divergences dans la détermination des cas, il faudrait mener des études de validation faisant appel à un examen direct des dossiers ou créer un registre global des AVC. 

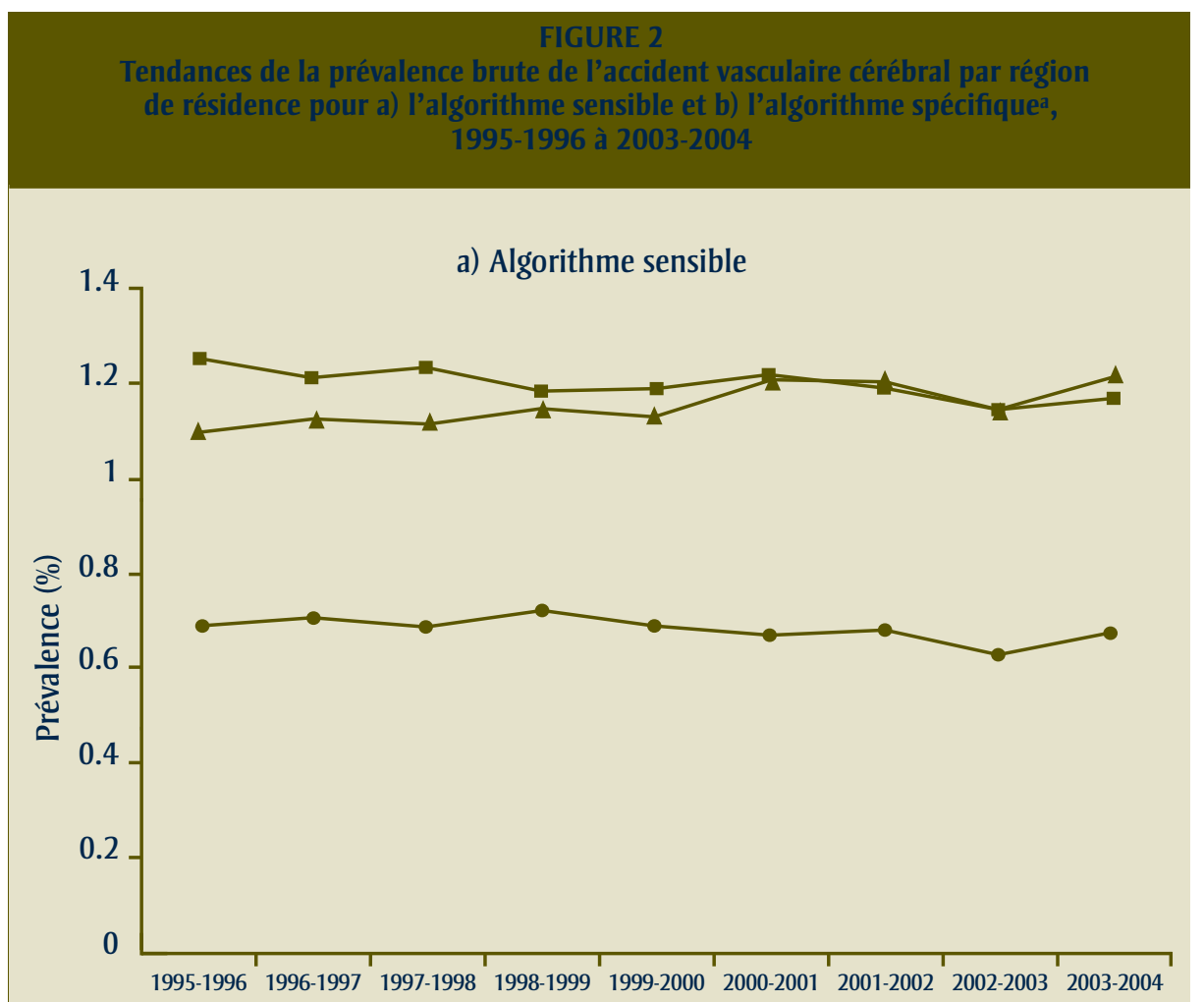

Année financière

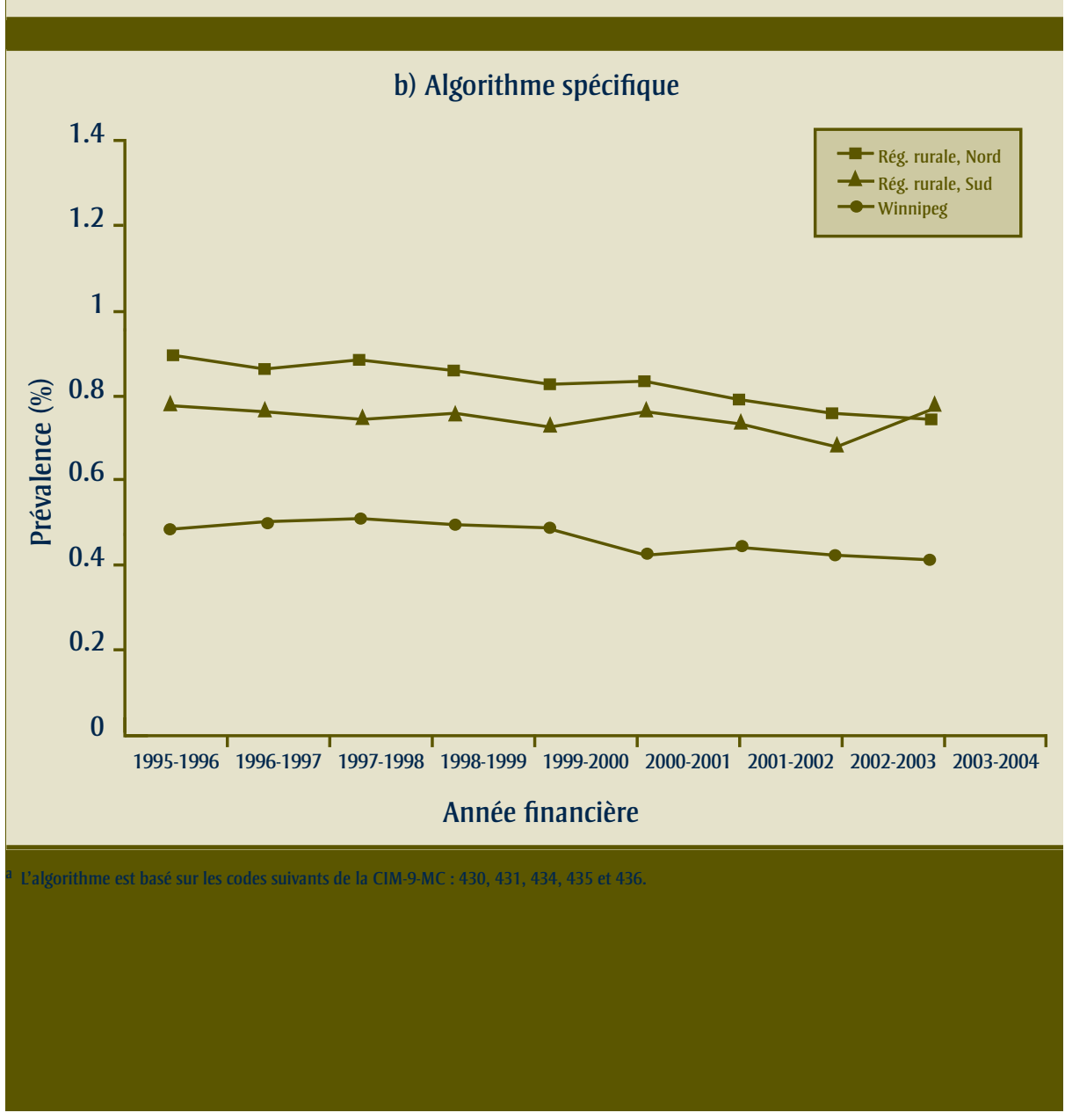

L'utilisation des demandes de paiement des médecins et des registres des médicaments de prescription, en plus des feuilles sommaires de départ des hôpitaux, a permis d'accroître le nombre total de cas d'AVC identifiés à partir des données administratives du Manitoba. En fait, l'inclusion des données relatives aux demandes de paiement des médecins a eu pour effet, à elle seule, de presque doubler le nombre de cas identifiés. Le recours aux registres des médicaments de prescription combinés aux demandes de paiement des médecins s'est soldé par l'identification d'un nombre restreint, mais croissant, de cas au fil du temps.

Le nombre total de cas d'AVC identifiés à partir des données administratives du Manitoba n'a guère changé dans le temps, bien que le nombre total de cas identifiés à partir des données des hôpitaux ait diminué. Cette observation pourrait avoir des incidences importantes sur les futures études de surveillance des AVC. Le recours à une unique source de données administratives pourrait se traduire par une image trompeuse de l'évolution de la prévalence de l'AVC dans le temps.

Les analyses des données annuelles sur l'AVC ont fait ressortir des variations significatives à la fois dans les groupes de revenu et les régions géographiques du Manitoba, même après ajustement pour tenir compte des différences sur le plan de l'âge et du sexe. Les analyses de tendances ont montré que la prévalence de l'AVC diminuait légèrement dans les groupes âgés comparativement au groupe le plus jeune, mais augmentait légèrement dans les régions urbaines et les régions rurales du Sud comparativement à la région du Nord. Les résultats de notre étude concordent dans une large mesure avec ceux d'autres études épidémiologiques, notamment l'étude de Framingham, qui ont mis en évidence une plus forte prévalence de l'AVC chez les hommes que chez les femmes, et un fardeau lié aux AVC qui est largement inchangé dans le temps ${ }^{4}$. Ces résultats ont des répercussions importantes sur le traitement de l'AVC, la perte d'autonomie à la suite de l'AVC, la réadaptation et l'élaboration de programmes ciblés de prévention de l'AVC. Ainsi, le traitement thrombolytique dans le groupe âgé se solde par une mortalité plus 
élevée, quoique ce groupe d'âge affiche une mortalité plus élevée que les groupes d'âge plus jeunes, même en l'absence de rt-PA ${ }^{30,31}$.

Cette étude présente certaines limites. Nous avons estimé la prévalence annuelle de l'AVC, mais l'incidence n'a pas été examinée. Il est cependant possible d'estimer le taux d'incidence de l'AVC en utilisant la pente de la tendance de la prévalence. La stabilité des estimations de la prévalence dans le temps donne à penser que l'incidence demeure relativement constante au Manitoba. Pour avoir un tableau complet de l'incidence, il faudrait toutefois utiliser les statistiques provinciales de l'état civil, en plus des données des hôpitaux et des médecins et celles relatives aux médicaments de prescription. Comme toutes les études épidémiologiques, l'examen de la détermination des cas d'AVC à partir des bases de données administratives représente un « instantané » des événements, qui comporte une erreur d'estimation implicite. Si l'erreur d'estimation n'a pas été quantifiée dans la présente étude, elle a toutefois été estimée de manière approximative par la présentation des résultats obtenus à l'aide de l'algorithme sensible et des algorithmes spécifiques, résultats qui représentent probablement les limites supérieure et inférieure de la prévalence de l'AVC.

Les données portent sur tous les résidants du Manitoba qui sont couverts par l'assurance-maladie de cette province au cours d'une année. Nous n'avons pas tenté d'exclure les résidants qui n'étaient que partiellement couverts au cours de l'année financière - en raison d'une migration à l'intérieur ou à l'extérieur de la province - et qui, par conséquent, pouvaient présenter une plus faible probabilité de satisfaire aux critères fixés aux fins de l'identification des cas d'AVC. En revanche, l'inclusion des résidants qui n'étaient que partiellement couverts au cours de l'année financière en raison d'un décès signifie que les données constituent une meilleure estimation de l'ensemble des cas d'AVC au Manitoba, et non seulement de ceux ayant survécu à l'AVC.
$\mathrm{Au}$ cours d'une récente étude portant sur la transition entre le codage au moyen de la CIM-9 et celui au moyen de la CIM-10, on n’a observé aucune différence significative entre les deux systèmes de classification pour ce qui est de la détermination des cas d'AVC ou des facteurs de risque ${ }^{6}$. Il y aurait lieu cependant d'évaluer, à l'aide des données du Manitoba, l'effet du changement du système de codage sur la détermination des cas d'AVC à partir des départs des hôpitaux.

En conclusion, il est possible d'utiliser les données administratives pour la surveillance de la population d'un éventail d'affections chroniques, dont l'accident vasculaire cérébral. Les données administratives peuvent servir à décrire les variations sociodémographiques au niveau de la prévalence de l'AVC dans la population et à réaliser des études rétrospectives de l'évolution au fil du temps. Ces données constituent un outil rentable pour obtenir de l'information sur le fardeau lié à l'AVC dans la population et pour guider la prise de décision en matière de politiques de santé.

\section{Remerciements}

Les auteurs tiennent à remercier Santé et mode de vie sain Manitoba, qui leur a fourni les données dans le cadre du projet n 2004/05-01. Cette étude a été financée, en partie, au moyen d'une bourse de nouveau chercheur des IRSC. Les conclusions et les résultats sont ceux des auteurs, et aucune approbation officielle de Santé et mode de vie sain Manitoba n'est recherchée ou ne devrait être inférée. Les auteurs n’ont aucun conflit d'intérêts à déclarer.

\section{Références}

1. Statistique Canada. Principales causes de décès sélectionnées, selon le sexe, Ottawa, Statistique Canada, 1997.

2. Verbrugge L, Lepkowski JM, Imanaka Y. Comorbidity and its impact on disability. Milbank Q. 1989;67:450-484.
3. Dobkin B. The Clinical Science of Neurologic Rehabilitation, deuxième édition, New York, Oxford, 2003:375-376.

4. Wolf P. Epidemiology of stroke. Dans : Mohr J, Choi DW, Grotta JC, Weir B, Wolf PA, (dir.), Stroke Pathophysiology, Diagnosis, and Management, Philadelphia, Churchill Livingstone, 2004:13-34.

5. Roos L, Walld R, Uhanova $\mathrm{J}$, Bond $\mathrm{R}$. Physician visits, hospitalizations, and socioeconomic status: Ambulatory care sensitive conditions in a Canadian setting. Health Research and Educational Trust. 2005;10: 1167-1185.

6. Kokotailo R, Hill MD. Coding of stroke and stroke risk factors using International Classification of Disease, Revision 9 and 10. Stroke. 2005;36:1776-1781.

7. Lix L, Yogendran M, Burchill C, et al. Defining and Validating Chronic Disease: An Administrative Data Approach, Winnipeg, Manitoba Center for Health Policy, 2006.

8. Yiannakoulias N, Svenson LW, Hill MD, et al. Regional comparison of inpatient and outpatient patterns of cerebrovascular disease diagnosis in the province of Alberta. Chronic Diseases in Canada 2003;24:9-16.

9. Ostbye T, Levy AR, Mayo NE. Hospitalization and case fatality rates for subarachnoid hemorrhage in Canada from 1982 through 1991. Stroke. 1997;28:793-798.

10. Mayo N, Chockalingam A, Reeder BA, et al Surveillance for stroke in Canada. Health Reports. 1994;6:62-72

11. Lappala J, Virtamo J, Heinonen OP. Validation of stroke diagnosis in the National Hospital Discharge Register and the Register of Causes of Death in Finland. Euro J Epi. 1999;15:155-160.

12. Benesch C, Witter DM, Wilder MA, et al. Inaccuracy of the International Classification of Diseases (ICD-9-CM) in identifying the diagnosis of ischemic cerebrovascular disease. Neurology. 1997;49:660-664. 
13. Tirschwell D, Longstreth WT. Validating administrative data in stroke research. Stroke. 2002;33:2465-2470.

14. Rhys Willams G, Jiang JG, Matchar DB, et al. Incidence and occurrence of total (first-ever and recurrent) stroke. Stroke. 1999;30:2423-2528.

15. The Publication Committee for the Trial of ORG 10172 in Acute Stroke Treatment (TOAST) Investigators. Low molecular weight heparinoid, ORG 1072 (danaparoid) and outcome after acute stroke. JAMA. 1998;279:1265.

16. Adams H, Bendixen BH, Kapele LJ, et al. Classification of subtype of acute ischemic stroke: Definitions for use in a multicenter clinical trial. Stroke. 1993;24:35-41.

17. Kolominisky-Rabas P, Weber M, Gefeller O, et al. Epidemiology of ischemic stroke subtypes according to TOAST criteria, Incidence, recurrence, and long-term survival in ischemic stroke subtypes: A population-based study. Stroke. 2001; 32:2735-2740.

18. Leibson C, Naessens JM, Brown RD, et al. Accuracy of hospital discharge abstracts for identifying stroke. Stroke. 1994;25: 2348-2355.

19. Madans J, Reubens C, Rothwell S, et al. Differences in morbidity measures and risk factor identification using multiple data sources: The case of stroke. J Epidemiol Biostat. 1999;4:37-43.

20. Rector T, Wickstrom SL, Shah M, et al. Specificity and sensitivity of claims-based algorithms for identifying members of Medicare plus Choice health plans that have chronic medical conditions. Health Serv Resear. 2004;39:1839-1861.

21. Stroup D, Berlin JA, Morton SC, et al. Metaanalysis of observational studies in epidemiology - A proposal for reporting. JAMA. 2000;283:2008-2012.

22. Friedman L, Furberg CD, DeMets DL. Fundamentals of Clinical Trials, $3^{\mathrm{e}}$ édition, New York, Springer, 1998:313-317.
23. SAS Institute Inc. SAS/STAT User's Guide, version 9,1, Cary, NC, SAS Institute Inc., 2004.

24. Roos N, Mustard CA. Variation in health and health care use by socioeconomic status in Winnipeg, Canada: Does the system work well? Yes and No. Milbank Q. 1997;75:89-111.

25. Robinson R, Young KT, Roos L, et al. Estimating the burden of disease: Comparing administrative data and self-reports. Med Care. 1997;35:932-947.

26. Statistique Canada. Profils provinciaux et territoriaux, Manitoba, Ottawa, Statistique Canada, 2001.

27. Organisation mondiale de la Santé. WHO Collaborating Centre for Drug Statistics Methodology: ATC Classification Index with DDDs and Guidelines for ATC Classification and DDD Assignment, Oslo, Norvège, Norwegian Institute of Public Health, 2006.

28. McCulloch CE, Searle SR. Generalized, Linear, and Mixed Models, New York, Wiley, 2001.

29. Fitzmaurice GM, Laird NM, Ware JH. Applied Longitudinal Analysis, Hoboken, NJ, Wiley, 2004.

30. Engelter S, Reichart M, Sekoranja L, et al. Thrombolysis in stroke patients aged 80 years and older: Swiss survey of IV thrombolysis. Neurology. 2005;65:1795-1798.

31. Hemphill J, Lyden P. Stroke thrombolysis in the elderly: Risk or benefit. Neurology. 2005;65:1690-1691.

32. Ellekjaer $\mathrm{H}$, Holmein $\mathrm{J}$, Oystein $\mathrm{K}$, et al. Identification of incident stroke in Norway hospital discharge data compared with a population-based stroke register. Stroke. 1999;30:56-60.

33. Reker DM, Hamilton BB, Duncan PW, et al. Stroke: Who's counting what? J Rehabil Res Devel. 2001;38:281-289. 